\title{
A Search for Distant Satellites of Neptune
}

\author{
Michael J. I. Brown and Rachel L. Webster \\ School of Physics, University of Melbourne, Parkville, Vic. 3052, Australia \\ mbrown@physics.unimelb.edu.au \\ Received 1998 June 10, accepted 1998 August 20
}

\begin{abstract}
The recent discovery of two distant satellites of Uranus suggests that there could be similar bodies orbiting Neptune. Previous surveys for distant satellites of Neptune have had relatively bright magnitude limits $\left(B_{J} \simeq 20.5\right)$ and would have missed satellites with magnitudes and colours similar to the two recently discovered Uranian satellites $(R \sim 20.4$ and $R \sim 21.9)$. We have searched for satellites of Neptune to a limiting magnitude of $R \sim 21$ in a $0 \cdot 1 \square^{\circ}$ region centred on the planet. This search is up to $\sim 2$ magnitudes deeper than the previous wide field search for distant satellites by the UK Schmidt Telescope. Nereid was easily recovered by the search and no large variations of its magnitude were detected.
\end{abstract}

Keywords: planets and satellites: Neptune, Nereid

\section{Introduction}

The recent discovery of two distant satellites of Uranus (Gladman et al. 1997) suggests similar bodies could be orbiting Neptune. Several surveys have looked for distant satellites of Neptune (Kuiper 1949, 1961; Hogg et al. 1994) but all have had magnitude limits $B_{J}<20 \cdot 5$. A review of all previous surveys for satellites of Neptune is provided by Hogg et al. (1994). The most recent survey for distant satellites of Neptune by Hogg et al. (1994) used the UK Schmidt Telescope to search the entire Roche lobe of Neptune to $B_{J} \sim 20 \cdot 5$.

We have used the Mount Stromlo and Siding Spring Observatories' 40-inch telescope to search for distant satellites of Neptune. Our field of view was $0 \cdot 1 \square^{\circ}$ so it was not practical to survey the entire Roche lobe of Neptune which extends $\sim 1.5^{\circ}$ from the planet. Instead we concentrated on one field centred on Neptune to obtain the deepest images possible within the time constraints of the observing run (see next section). If Neptune had distant satellites at similar angular distances from the planet as the recently discovered Uranian moons $\left(\sim 7^{\prime}\right)$, they would have been within the field of view of our CCD.

As well as searching for distant satellites of Neptune, we also observed Neptune's distant moon Nereid. There has been considerable debate in the literature about the rotation of Nereid and the magnitude of its light curve (Schaefer \& Schaefer 1988a, 1988b; Bus \& Larson 1989; Williams, Jones \& Taylor 1991; Thomas, Veverka \& Helfenstein 1991; Dobrovolskis 1995; Buratti, Goguen \& Mosher 1997). Our observations are not a complete light curve of
Nereid though they are consistent with Nereid not having large magnitude changes.

\section{Observations and Analysis}

Two nights of observations were obtained on 30 September 1997 and 1 October 1997 with the Mount Stromlo and Siding Spring Observatories' 40-inch telescope. Our detector was a $2048 \times 2048$ thinned Tektronix CCD with a $0 \cdot 6^{\prime \prime}$ pixel scale. The primary purpose of the observing run was obtaining photometry of the South Galactic Pole (SGP) so observations of Neptune were restricted to an hour each night when the SGP was low in the sky. Weather conditions on the first night were reasonable with $\sim 2^{\prime \prime}$ seeing but on the second night thin cloud produced $\sim 3^{\prime \prime}$ seeing and $\sim 0.7$ magnitudes of extinction. Each CCD exposure was $400 \mathrm{~s}$ in the $R$ band and the total integration times on the two nights were $2800 \mathrm{~s}$ and $2000 \mathrm{~s}$.

Each image was bias subtracted and flatfielded using IRAF. Fluxes of several thousand stars in each field were then determined with SExtractor (Bertin \& Arnouts 1996) and extinction corrections were then determined by comparing object fluxes between images. The images were multiplied with IRAF's imarith routine to correct for extinction and then combined to produce two deep images (one for each night). SExtractor was then used to detect objects in each image and our own code was then used to search for objects moving with rates of motion within $12^{\prime \prime}$ per day of the rate of motion of Neptune. Images were also blinked to detect objects missed by the automated analysis and to detect any satellites close to Neptune with apparent motions 
$>12^{\prime \prime}$ per day relative to the position of the planet. Neptune and its moon Nereid were easily detected by the automated analysis and blinking but no new satellites were discovered. Triton was not detected as it was only $16^{\prime \prime}$ from Neptune and was lost in the scattered light from the planet.

Table 1. Detection efficiency

\begin{tabular}{cc}
\hline$R$ magnitude & $\begin{array}{c}\text { Percentage of moving } \\
\text { objects detected }\end{array}$ \\
\hline $19 \cdot 0$ & 78 \\
$20 \cdot 0$ & 66 \\
$20 \cdot 5$ & 59 \\
$21 \cdot 0$ & 50 \\
$21 \cdot 5$ & 42 \\
\hline
\end{tabular}

To determine the magnitude limit of the search, artificial objects were added to the data with IRAF's mkobject routine. The magnitudes of the artificial objects were calibrated with CCD images of Landolt Standards (Landolt 1992) taken on 30 September 1997. The same data analysis was used to search for artificial objects as real objects. The detection efficiency for the artificial objects is listed in Table 1. This search is $50 \%$ complete to a limiting magnitude of $R \sim 21$. The relatively low detection efficiency at bright magnitudes $(\sim 80 \%)$ is caused by the large number of background stars due to the low galactic latitude of Neptune $\left(-24^{\circ}\right)$ at the time of the observations. If we assume distant satellites of Neptune could have similar colours to Uranian satellites (S/1997 U2's colour is $B-R \sim 1 \cdot 5$, Gladman et al. 1997), then our search is up to $\sim 2$ magnitudes deeper than the previous survey by Hogg et al. (1994).

\section{Nereid}

There has been considerable discussion in the literature about the rotation of Nereid and the amplitude of its light curve (Schaefer \& Schaefer 1988a, 1988b; Bus \& Larson 1989; Williams, Jones \& Taylor 1991; Thomas, Veverka \& Helfenstein 1991; Dobrovolskis 1995; Buratti, Goguen \& Mosher 1997). A summary of these papers is provided by Buratti, Goguen \& Mosher (1997). While our $12 \times 400 \mathrm{~s}$ $R$-band images of Nereid only measure the light curve for 1 hour on each night, a large magnitude change would be strong evidence of a large amplitude $(\Delta m a g>1)$ light curve similar to those reported by Schaefer \& Schaefer (1988a, 1988b) and Williams, Jones \& Taylor (1991).

The observations and data reduction are described in the previous section. Each $400 \mathrm{~s} R$-band image was used (rather than the combined images) so any rapid changes of the magnitude of Nereid could be easily detected. SExtractor (Bertin \& Arnouts 1996) was used to obtain the photometry of Nereid and this photometry is summarised in Table 2 .
Table 2. Nereid photometry

\begin{tabular}{lcc}
\hline UT date & UT time & $\begin{array}{c}R \text { band magnitude } \\
\text { and } 3 \sigma \text { error }\end{array}$ \\
\hline 30 Sep 1997 & $9: 40$ & $18 \cdot 98 \pm 0 \cdot 07$ \\
30 Sep 1997 & $9: 52$ & $18 \cdot 98 \pm 0 \cdot 07$ \\
30 Sep 1997 & $10: 04$ & $18 \cdot 95 \pm 0 \cdot 07$ \\
30 Sep 1997 & $10: 16$ & $18 \cdot 92 \pm 0 \cdot 07$ \\
30 Sep 1997 & $10: 28$ & $18 \cdot 98 \pm 0 \cdot 07$ \\
30 Sep 1997 & $10: 40$ & $18 \cdot 94 \pm 0 \cdot 07$ \\
30 Sep 1997 & $10: 52$ & $18 \cdot 91 \pm 0 \cdot 07$ \\
30 Sep 1997 & Average & $18 \cdot 95 \pm 0 \cdot 03$ \\
1 Oct 1997 & $10: 03$ & $19 \cdot 05 \pm 0 \cdot 09$ \\
1 Oct 1997 & $10: 14$ & $19 \cdot 05 \pm 0 \cdot 09$ \\
1 Oct 1997 & $10: 40$ & $19 \cdot 01 \pm 0 \cdot 07$ \\
1 Oct 1997 & $10: 52$ & $19 \cdot 05 \pm 0 \cdot 07$ \\
1 Oct 1997 & $11: 04$ & $19 \cdot 04 \pm 0 \cdot 06$ \\
1 Oct 1997 & Average & $19 \cdot 04 \pm 0 \cdot 04$ \\
\hline
\end{tabular}

Table 2 shows that the magnitude of Nereid does not significantly change on either night of observation. A $0 \cdot 09 \pm 0 \cdot 05$ magnitude increase is seen between the two nights but the error estimate for this magnitude change does not include systematic errors which may have varied between the two nights of observations.

It should also be noted that Nereid is in a busy field during the observations and this could introduce a bias into the magnitude estimates. Our measured light curve for Nereid is consistent with observations by Thomas, Veverka \& Helfenstein (1991) and Buratti, Goguen \& Mosher (1997) who also found no large variations of Nereid's magnitude. Our $R$-band magnitudes are also similar to those reported by Buratti, Goguen \& Mosher (1997). We cannot rule out a large amplitude light curve for Nereid, however, our observations are consistent with Nereid having a light curve with $\Delta m a g<0 \cdot 1$.

\section{Conclusions}

We have completed a search for distant satellites of Neptune in a $0 \cdot 1 \square^{\circ}$ region centred on the planet. Nereid was easily recovered but no new satellites were detected to the limiting magnitude of $R \sim 21$. Our limiting magnitude is up to $\sim 2$ magnitudes deeper than the previous search for distant satellites of Neptune by Hogg et al. (1994). If a satellite with an apparent $R$ magnitude similar to Uranian moon S/1997 U2 had been within the field of view of our observations, there is a $\sim 60 \%$ probability that it would have been detected. Photometry of Nereid was obtained on both nights of observations and no large magnitude variations were detected. A dedicated observing run with the $8 \mathrm{k} \times 8 \mathrm{k}$ CCD Wide Field Imager being constructed for the Mount Stromlo and Siding Spring Observatories' 40-inch telescope should allow most of the Roche lobe of Neptune to be searched to $R \sim 22.5$ and improve constraints on the light curve of Nereid. 


\section{References}

Bertin, E., \& Arnouts, S. 1996, A\&AS, 117, 393

Buratti, B. J., Goguen, J. D., \& Mosher, J. A. 1997, Icarus, 126,225

Bus, E. S., \& Larson, S. 1989, BAAS, 21, 982

Dobrovolskis, A. R. 1995, Icarus, 118, 181

Gladman, B. J., Nicholson, P. D., Burns, J. A., \& Kavelaars, J. J. 1997, IAUC 6763

Hogg, D., Jackson, C., Żytkow, A. N., Irwin, M., \& Webster, R. 1994, Icarus, 107, 304
Kuiper, G. P. 1949, PASP, 61, 175

Kuiper, G. P. 1961, in Planets and Satellites, eds G. P. Kuiper \& B. M. Middlehurst (Univ. Chicago Press), p. 575

Landolt, A. U. 1992, AJ, 104, 340

Schaefer, M. W., \& Schaefer, B. E. 1988a, Nature, 333, 436

Schaefer, M. W., \& Schaefer, B. E. 1988b, BAAS, 27, 1167

Thomas, P., Veverka, J., \& Helfenstein, P. 1991, J. Geophys. Res., 96, 19253

Williams, I. P., Jones, D. H. P., \& Taylor, D. B. 1991, MNRAS, 250, 1 